\title{
Visual Field Map Clusters in Macaque Extrastriate Visual Cortex
}

\author{
Hauke Kolster, ${ }^{1,2}$ Joseph B. Mandeville, ${ }^{1,2}$ John T. Arsenault, ${ }^{1}$ Leeland B. Ekstrom, ${ }^{1,3,4}$ Lawrence L. Wald, ${ }^{1,2,3}$ and \\ Wim Vanduffel ${ }^{1,2,5}$ \\ ${ }^{1}$ Athinoula A. Martinos Center for Biomedical Imaging, Massachusetts General Hospital, Charlestown, Massachusetts 02129, ${ }^{2}$ Department of Radiology, \\ Harvard Medical School, Charlestown, Massachusetts 02129, ${ }^{3}$ Harvard-Massachusetts Institute of Technology Division of Health Sciences and Technology, \\ Cambridge, Massachusetts 02139, ${ }^{4}$ Department of Nuclear Science and Engineering, Massachusetts Institute of Technology, Cambridge, Massachusetts \\ 02139, and 5aboratorium voor Neuro- en Psychofysiologie, Katholieke Universiteit Leuven Medical School, Campus Gasthuisberg, 3000 Leuven, Belgium
}

The macaque visual cortex contains $>30$ different functional visual areas, yet surprisingly little is known about the underlying organizational principles that structure its components into a complete "visual" unit. A recent model of visual cortical organization in humans suggests that visual field maps are organized as clusters. Clusters minimize axonal connections between individual field maps that represent common visual percepts, with different clusters thought to carry out different functions. Experimental support for this hypothesis, however, is lacking in macaques, leaving open the question of whether it is unique to humans or a more general model for primate vision. Here we show, using high-resolution blood oxygen level-dependent functional magnetic resonance imaging data in the awake monkey at $7 \mathrm{~T}$, that the middle temporal area (area MT/V5) and its neighbors are organized as a cluster with a common foveal representation and a circular eccentricity map. This novel view on the functional topography of area MT/V5 and satellites indicates that field map clusters are evolutionarily preserved and may be a fundamental organizational principle of the Old World primate visual cortex.

\section{Introduction}

Following the seminal work of Ungerleider and Mishkin (1982) and Goodale and Milner (1992) that resulted in the concept of dorsal and ventral visual streams, several hypotheses about the functional organization of visual cortex have been proposed (Felleman and Van Essen, 1991; Allman, 1999; Hasson et al., 2002; Wandell et al., 2005). General agreement exists that a successful model should conform to the principle of minimum wiring length (Chklovskii and Koulakov, 2004). In other words, the axonal length for communication between processing modules for specific perceptual functions should be kept minimal (Mitchison, 1991).

One group has suggested that maps in ventral occipital cortex are organized according to a central versus peripheral visual field bias (Hasson et al., 2002). Such an organizing principle, however,

\footnotetext{
Received Jan. 31, 2009; revised April 17, 2009; accepted April 19, 2009.

This work received support from a Natural Sciences and Engineering Research Council of Canada postgraduate scholarship, the Human Frontier Science Program Organization, Geneeskundige Stichting Koningin Elisabeth, Interuniversity Attraction Pole 5/04, Excellentie Financiering EF/05/014, Geconcerteerde Onderzoeksactie GOA/10/019, Fonds voor Wetenschappelijk Onderzoek Grants G.0622.08 and G.0593.09, National Institute of Biomedical Imaging and Bioengineering Grants R01-EB00790 and R01EB006847, and National Science Foundation Grant BCS-0745436. The Martinos Center is supported by the National Center for Research Resources Grant P41RR14075 and the Menta Illness and Neuroscience Discovery Institute. We thank H. Deng for animal training and care, R. Tootell, A. Potthast, and B. Rosen for advice and support, and L. Ungerleider, B. Wandell, G. Orban, K. Nelissen, and J. Polimeni for valuable comments on the manuscript.

Correspondence should be addressed to Wim Vanduffel, Athinoula A. Martinos Center for Biomedical Imaging, Massachusetts General Hospital, Charlestown, MA 02129. E-mail: wim@nmr.mgh.harvard.edu.

H. Kolster's and J. T. Arsenault's present address: Laboratorium voor Neurofysiologie en Psychofysiologie, Katholieke Universiteit Leuven Medical School, Campus Gasthuisberg, 3000 Leuven, Belgium.

D0I:10.1523/JNEUROSCI.0518-09.2009

Copyright $\odot 2009$ Society for Neuroscience $\quad$ 0270-6474/09/297031-09\$15.00/0
}

cannot provide a complete explanation for the retinotopic organization of extrastriate cortex, since it would render polar angle representations unnecessary. This is in contradiction with monkey (Brewer et al., 2002; Fize et al., 2003) and human (Brewer et al., 2005; Larsson and Heeger, 2006; Saygin and Sereno, 2008) imaging data revealing polar angle maps in several higherorder visual areas. Moreover, an eccentricity-bias model would apply mainly to object-selective regions, where foveal overrepresentation is most relevant. Therefore, it would be difficult to generalize such a model to a unifying principle of cortical functional organization.

An alternative model poses that visual field maps are organized as clusters (Wandell et al., 2005). Within a cluster, several maps share a common foveal representation, surrounded by a circular eccentricity map, with polar angle representations sprouting radially from the center. Thus, a cluster would appear as a "pinwheel," resembling orientation pinwheels in V1 (Bonhoeffer and Grinvald, 1991), although on a much larger spatial scale. A pinwheel-like functional layout may be the most efficient manner to minimize wiring length between processing modules that require strong functional interactions, regardless of their spatial scale. While elegant, several problems exist with this hypothesis. Although a number of foveal representations have been identified in human extrastriate visual cortex beyond V4, little evidence exists that multiple field maps share these representations [but see Brewer et al. (2005)]. More importantly for a general model, clusters should be present not only in ventral but also in dorsal-stream visual areas. Moreover, clusters should be evolutionarily preserved and therefore exist in Old World monkeys. Yet, despite $>40$ years of research, no evidence of field map clus- 
ters in macaque extrastriate cortex currently exists. Finally, a cluster should group field maps that share common functionalities - a key assumption of the model that has not yet been demonstrated.

Using high-resolution functional magnetic resonance imaging (fMRI), we tested the prediction that field map clusters do exist in monkey visual cortex. More specifically, we hypothesized that a group of dorsal stream areas within the superior temporal sulcus (STS) that are all specialized in visual motion processing (Komatsu and Wurtz, 1988) should be organized as a cluster. The resulting fMRI data challenge our current understanding of the functional topography of middle temporal area (area MT/V5) and surrounding areas, as will be detailed below.

\section{Materials and Methods}

Animals and behavior. All procedures were approved by the Massachusetts General Hospital Subcommittee on Research Animal Care (Protocol \#2003N000338) and are in accordance with National Institutes of Health guidelines for the care and use of laboratory animals. Two male rhesus monkeys (Macaca mulatta; $\mathrm{CH}$ and TO, 5-7 kg, 4-5 years old) were prepared for fMRI as previously described (Vanduffel et al., 2001) and trained for a passive fixation task. General behavioral and scanning procedures (except for the use of the $7 \mathrm{~T}$ scanner and parallel imaging) have been described previously (Vanduffel et al., 2001, 2002; Fize et al., 2003; Nelissen et al., 2006). The monkeys sat in a sphinx position, and their heads were fixed with a head post while performing a visual fixation task.

Anatomical MRI acquisition. High resolution, T1-weighted anatomical images were collected on a whole-body $3 \mathrm{~T}$ scanner for the overlay of functional analyses. Under ketamine-xylazine anesthesia, an magnetization-prepared rapid-acquisition gradient echo (MPRAGE) sequence (178 sagittal slices, $256 \times 256$ in-plane matrix, repetition time $(\mathrm{TR})=2.5 \mathrm{~s}$, echo time $(\mathrm{TE})=4.35 \mathrm{~ms}$, inversion time $=1100 \mathrm{~ms}, 0.35$ $\mathrm{mm}$ isotropic voxels, flip angle $=8^{\circ}$ ) was used to obtain nine whole-brain volumes, which were averaged together to improve the signal-to-noise ratio (Fig. $1 A$; supplemental Fig. S1 $A$, available at www.jneurosci.org as supplemental material). A single radial transmit-receive surface coil $(12.5 \mathrm{~cm}$ diameter) was used.

Functional MRI acquisition. We significantly adapted existing monkey fMRI methods (Vanduffel et al., 2001) to overcome problems inherent to body motion - which are especially prominent at high magnetic field strengths (Goense et al., 2008). Functional scanning of the awake monkeys was performed in a horizontal 7 T MRI scanner equipped with a 36 $\mathrm{cm}$ inner-diameter head gradient set (AC88, maximum strength: 80 $\mathrm{mT} / \mathrm{m}$; maximum slew rate: $800 \mathrm{~T} / \mathrm{m} / \mathrm{s}$ ), and second- and third-order resistive shim coils. fMRI data were collected as raw blood oxygen leveldependent (BOLD) images and were acquired with an isotropic spatial resolution of $0.75 \times 0.75 \times 0.75 \mathrm{~mm}^{3}\left(0.42 \mathrm{~mm}^{3}\right)$. Four- and eightchannel phased array receive coils, with, respectively, $5 \mathrm{~cm}$ and $3.5 \mathrm{~cm}$ coil diameters, were custom built to fit juvenile rhesus monkeys. A singleshot, T2*-weighted, gradient-echo echo-planar image (GE-EPI) sequence was used with TR/TE $=2000 / 19 \mathrm{~ms}$, an echo spacing of $0.29 \mathrm{~ms}$, a field of view of $72 \mathrm{~mm}$, and 36-42 horizontal slices centered over the dorsal part of the cerebrum (Fig. $1 B$; supplemental Fig. S1 $B$, available at www.jneurosci.org as supplemental material). These parameters resulted in average signal-to-noise ratio values of 35 and 80 for the four- and eight-channel coils, respectively. Within each TR, half of the $k$-space volume was acquired by alternating between odd and even lines of the full $k$-space volume. We acquired 8192 functional images in $\mathrm{CH}$ and 6144 images in TO. In these experiments, we typically observed BOLD signal changes in the visual cortex of $\sim 4 \%$ (supplemental Fig. S2, available at www.jneurosci.org as supplemental material). The resulting raw EPI images were corrected for lowest-order off-resonance effects and aligned with respect to the gradient-recalled-echo reference images before performing a SENSE (sensitivity encoding) image reconstruction (Pruessmann et al., 1999). Residual N/2 artifacts in the reconstructed images were removed using an algorithm based on the UNFOLD method (Madore et al., 1999) and were further corrected for higher-order distortions using a nonrigid slice-by-slice distortion correction.

Visual stimuli. Retinotopic stimuli were presented as expanding rings and rotating wedges in four cycles per run with each run lasting $64 \mathrm{~s}$ and typically 32 runs per session divided between the two stimuli. The stimuli were projected at $1024 \times 768$ resolution and $60 \mathrm{~Hz}$ refresh rate from a liquid crystal display projector onto a translucent screen $52 \mathrm{~cm}$ from the eyes. Successful fixation behavior in both animals was better than $90 \%$ within a $1^{\circ}$ fixation radius as measured at $120 \mathrm{~Hz}$ with an infrared-based eye-tracking system. The stimuli covered between 1 and $12^{\circ}$ eccentricity. We used monochromatic, $6 \mathrm{~Hz}$ counterphasing high-contrast checkerboard stimuli (wedges: 45 degrees wide, 16 checkers $/ 360^{\circ}$ and annuli: 24 checkers $/ 360^{\circ}$ ). The radial size and spatial frequency of the eccentricity rings were scaled according to a $\log (r)$ law, as was the radial size of the checkers within the eccentricity rings and polar angle wedges and kept close to an aspect ratio of 1:1. The sizes of both the polar angle wedge in the azimuthal direction and the expanding circles in the radial direction were designed to illuminate points on the screen for $8 \mathrm{~s}$, after which the hemodynamic response reaches its maximum (Leite and Mandeville, 2006). This procedure maximizes the time between two concurrent activations in which the response can reach the baseline before being activated in the following cycle. The initial $8 \mathrm{~s}$ of each run $(4 \mathrm{TR})$ are used to reach equilibrium in the hemodynamic response. Although no data were recorded during this time, we presented the stimulus of the last four TRs of a cycle to simulate a continuous cycle at the start of each run. To create a smooth transition and to avoid a sudden jump of the rings from largest to smallest eccentricity at the end of each cycle, we masked the stimulus at the lowest and highest eccentricity positions. Here, the stimulus would gradually disappear and then again appear from behind the masks. We further presented a blank screen for one TR as a transition from high to low eccentricities. Each run consisted of 4 cycles lasting $256 \mathrm{~s}$. Between 24 and 32 runs were acquired per session separated by resting periods of 4-7 $\mathrm{min}$. The resting periods were needed to prevent overheating of the gradient coils and also helped to improve the performance of the monkeys during long scan sessions.

The effect of the receptive field size is seen as a broadening of the 

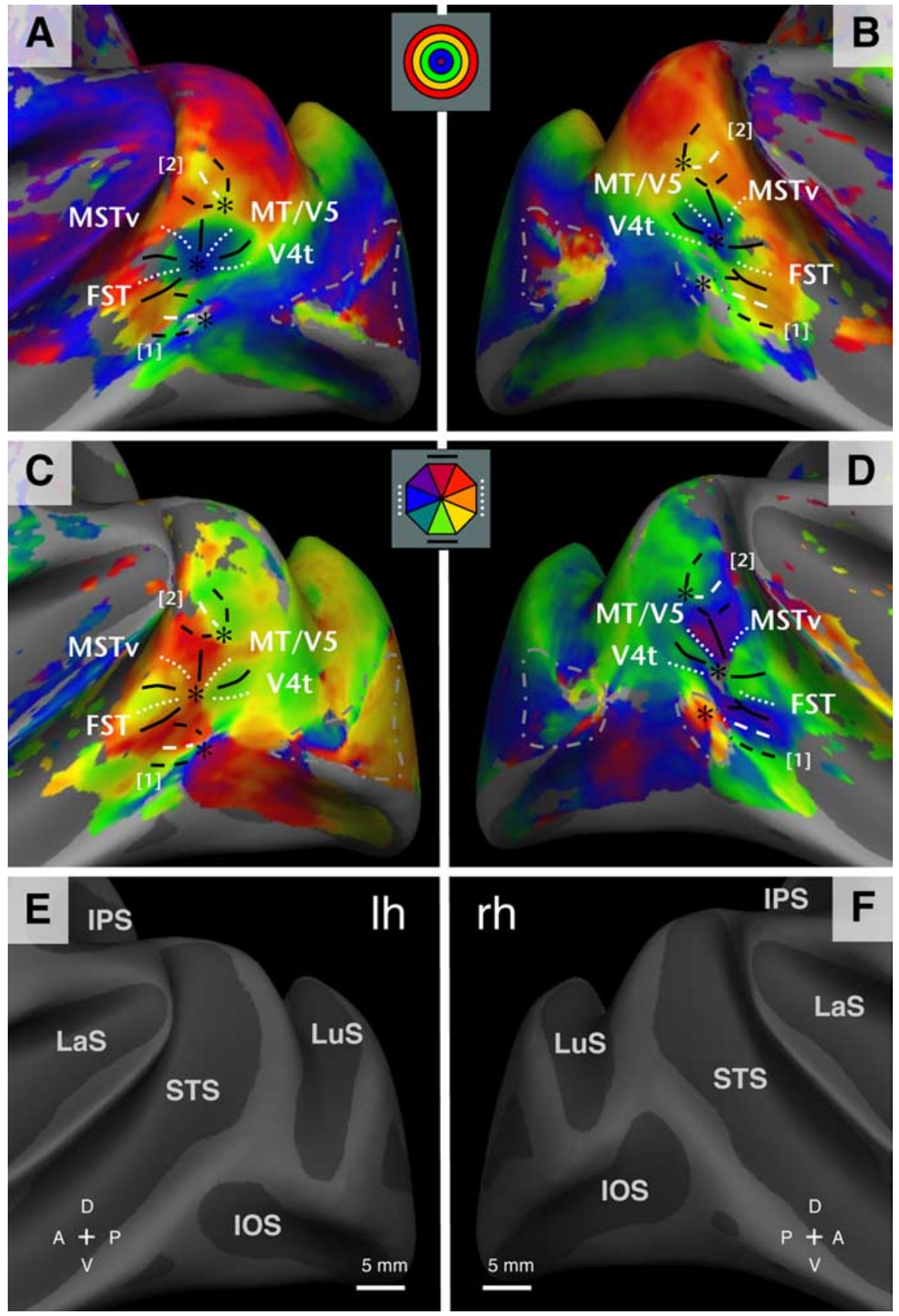

Figure 2. Retinotopy of eccentricity and polar angle representations in caudal macaque STS of subject $C H . A, B$, Eccentricity maps of the left hemisphere $(\boldsymbol{A})$ and right hemisphere $(\boldsymbol{B}) . \boldsymbol{C}, \boldsymbol{D}$, Polar angle maps of the left hemisphere $(\boldsymbol{C})$ and right hemisphere (D). $\boldsymbol{E}, \boldsymbol{F}$, Inflated surface reconstructions of the left hemisphere $(\boldsymbol{E})$ and right hemisphere $(\boldsymbol{F})$. The representation of central to peripheral eccentricities is coded using blue-green-yellow-red, respectively. The colors red, orange/dark blue, and green represent the upper, middle, and lower parts of the contralateral visual field, respectively. An asterisk indicates each representation of the center of gaze. White dotted and black solid lines indicate horizontal and vertical meridian representations, respectively, within the MT/V5 field map cluster. The dashed white and black lines indicate horizontal and vertical meridians in field maps ventral [1] and dorsal [2] to the MT/V5 cluster. The gray dashed-dotted line indicates the central visual representation for early areas, which were not activated by the stimuli in this study. The curvature of the cortex is shown using light and dark gray to signify convexity (gyri) or concavity (sulci). IOS, Inferior occipital sulcus; LuS, lunate sulcus; LaS, lateral sulcus; IPS, intraparietal sulcus; A, anterior; $P$, posterior; $D$, dorsal; $V$, ventral; $M$, medial; $L$, lateral.

response function as demonstrated in supplemental Fig. S2 (available at www.jneurosci.org as supplemental material). Here, the average BOLD response is shown for an individual voxel within area V1 (small receptive field size) and area V4 (larger receptive field size). While there is a clear gap between the response peaks in V1, the signal in V4 just reaches baseline before rising again, indicating that the width of the response peaks in V4 is increased compared with the response peaks in V1 (Sereno et al., 1995; Smith et al., 2001; Dumoulin and Wandell, 2008).

Data analysis. All runs of the eccentricity and polar angle experiments acquired within one session were averaged into one data file with a length of 128 time points. FREESURFER tools (Fischl et al., 1999) were then used to register the EPI volumes to the anatomical volumes. We independently registered the left and right functional volumes to the anatomical volume because it improved the registration procedure for the left and right STS. During registration, we focused on MT/V5 and neighboring areas and paid less attention to areas not discussed in the present manuscript. It is noteworthy that compared with the posterior portion of the STS, susceptibility artifacts are more pronounced at the ventral occipital pole. This might have led to slightly distorted activity maps in ventral portions of $\mathrm{V} 1, \mathrm{~V} 2$, and $\mathrm{V} 3$ as exemplified by the apparent underrepresentation of the upper quadrant in these early visual areas. At the level of the STS, on which this study is entirely focused, the registration of the functional with anatomical images was more straightforward; hence, the retinotopic data could be mapped accurately on the relevant reconstructed portion of the STS (see Figs. 1, 2).

Each voxel in the functional volumes was analyzed for phase-shift information, which is related to degrees of eccentricity or polar angle. Maps of the phase-shift information and corresponding $p$ values were then calculated and registered to the anatomical volumes. The phaseshift data were projected on several surfaces that represent cortical layers parallel to the pial surface. We chose layers at distances of 0.1-0.9 times the local gray matter thickness above the pial surface in steps of 0.1 . The resulting maps that arose from the different cortical depths were averaged across the cortical thickness and painted onto the inflated and flattened surfaces. Further smoothing with a kernel size of 1 voxel was applied on the surface during the paint process. An uncorrected $p$ value of $10^{-3}$ was used as a threshold for all experiments. Field sign maps were then calculated based on the individual maps for eccentricity and polar angle (Sereno et al., 1995).

Assignment of field maps and meridians. We used contour plots to derive isoeccentricity lines from eccentricity maps at different levels of eccentricities (supplemental Figs. $\mathrm{S} 3 B, \mathrm{~S} 4 B$, $\mathrm{S} 5 B, \mathrm{~S} 6 B$, available at www.jneurosci.org as supplemental material). For this purpose, we set the image brightness to $35 \%$ and the contrast to $100 \%$. Vertical and horizontal meridians were assigned based on information from both the polar angle maps and the field sign maps (supplemental Figs. S3E, S4E, S5E, S6E, available at www.jneurosci.org as supplemental material). The field sign map allows for identification of individual visual areas based on equal field sign, with a change of field sign indicating the border between areas. The assignment of meridians requires a coincidence between the location of a specific event on the field sign map and the polar angle map. In case of a hemifield, areal borders are defined by vertical meridians. The vertical meridian must represent a transition in the field sign map and 
coincide with the color for a vertical meridian (green or purple). The horizontal meridian must represent the centroid of a mirror or nomirror region and coincide with the color for a horizontal meridian (blue or orange). In case of a quarter field, e.g., area V4t, both vertical and horizontal meridians represent areal borders. Here, the horizontal meridian represents a transition between regions in the field sign map and a blue color in the polar angle map.

Phase calibration. We calibrated the color scale for the polar angle distribution for subject $\mathrm{CH}$ using an independent experiment presenting fixed stimuli of the vertical ( $\pm 6^{\circ}$ polar angle) and horizontal $\left( \pm 3^{\circ}\right.$ polar angle $)$ meridians using the same checkerboard design. A comparison of the representation of the vertical versus the horizontal meridian provided the calibration of the color wheel used in the phaseencoded experiments.

Repeatability. We performed test and retest measurements of the phase-encoded retinotopy experiments in both subjects. In subject $\mathrm{CH}$, we used first the four-channel and later the eight-channel phased-array receive coil in two sessions separated by 5.5 months. In subject $\mathrm{TO}$, the eight-channel phased-array receive coil was used in two sessions separated by 2.5 months.

\section{Results}

In two awake, fixating monkeys $(\mathrm{CH}$ and TO), we performed a phase-encoded retinotopic mapping experiment at $7 \mathrm{~T}$ (Sereno et al., 1995) using stimuli with eccentricities between 1 and $12^{\circ}$ and a full variation of polar angle. As predicted from single unit and anatomical tract tracing studies (Desimone and Ungerleider, 1986; Mikami et al., 1986; Maunsell and Van Essen, 1987; Komatsu and Wurtz, 1988; Tanaka et al., 1993), we identified a foveal representation near the fundus of the posterior portion of the STS [Fig. $2 A, B$ (monkey $\mathrm{CH}$ ); supplemental Fig. S7 $A, B$, available at www.jneurosci.org as supplemental material (monkey TO)] that is anatomically distinct from the foveal representation in early visual areas (V1, V2, V3, V3A, and V4). In addition, the fMRI data revealed (1) a continuous eccentricity map spanning a three-quarter circle surrounding this singular foveal representation (Fig. 2A,B; supplemental Fig. $\mathrm{S} 7 A, B$, available at www.jneurosci.org as supplemental material), (2) a polar angle map showing eight alternating representations of horizontal and vertical meridians (Fig. 2C,D; supplemental Fig. S7C,D, available at www.jneurosci.org as supplemental material), and (3) a field sign map indicating the existence of four individual areas joined at this foveal representation (supplemental Figs. S3E, S4E, S5E, S6E, available at www.jneurosci.org as supplemental material). Thus, one contralateral quarter-field plus three complete contralateral hemifield representations surrounding a common foveal representation and sharing a continuous circular eccentricity map in this portion of the STS are found consistently in all four hemispheres.

Figure $3 \mathrm{~A}$ shows the polar angle phase variation as a function
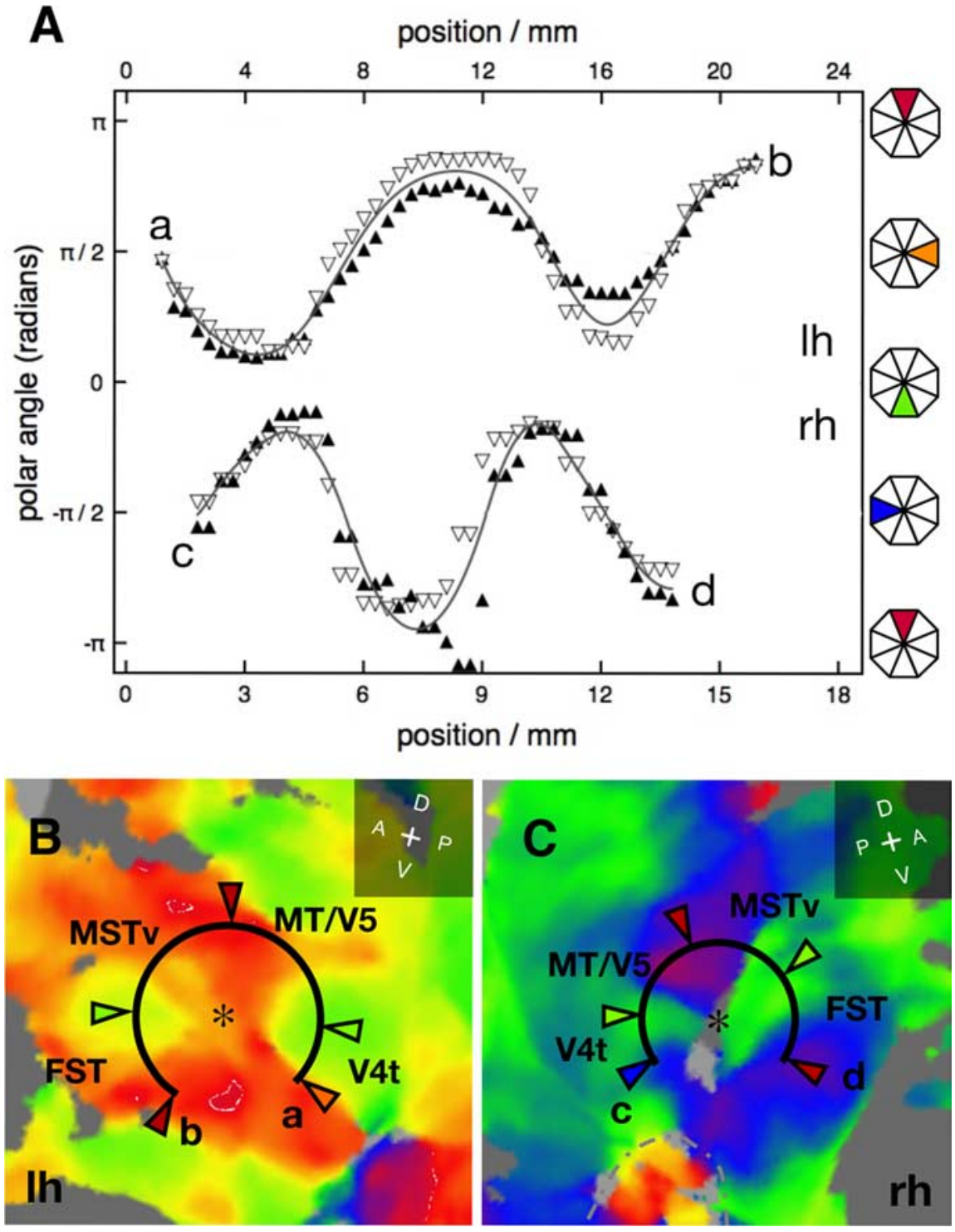

Figure 3. Polar angle phase variation within the MT/V5 cluster; subject $\mathrm{CH}$. $\boldsymbol{A}$, Polar angle phase as a function of distance along a circle within the MT/V5 cluster $(\mathrm{CH})$; top, from a to $b$ for the left hemisphere $(\boldsymbol{B})$; bottom, from c to $\mathrm{d}$ for the right hemisphere $(\boldsymbol{C})$. Open symbols (data from supplemental Figs. S4D, S5D, available at www.jneurosci.org as supplemental material) and closed retest data, respectively. Correlation between datasets: right hemisphere (bottom) $R^{2}=0.87, p=1 \times 10^{-23}$ and left hemisphere (top) $R^{2}=0.71, p=6 \times 10^{-12}$. The line represents a spline fit to the average of the two datasets.

of distance along a curved line surrounding the foveal MT/V5 representation based on a detailed analysis of the polar angle data. We subsampled the polar angle data, interpolated it onto a Cartesian grid of $0.5 \mathrm{~mm}$, and smoothed it with a Gaussian kernel of $0.5 \mathrm{~mm}$ to restore the initial resolution of $0.75 \mathrm{~mm}$. We selected grid points that coincided with points along a preselected circular path on the grid for display. Both test and retest data were projected onto the same surfaces, and we used the same circular path on the Cartesian grid for both datasets.

The data confirms multiple phase reversals, thereby corroborating the existence of one contralateral quarter-field and three contralateral hemifield representations. The individual field maps are separated by representations of the upper and lower vertical meridians, as indicated by the color-coded wedges in Figure $3 A$. Figure 3, $B$ and $C$, shows enlarged versions of the polar angle maps from the posterior STS of the left and right hemi- 

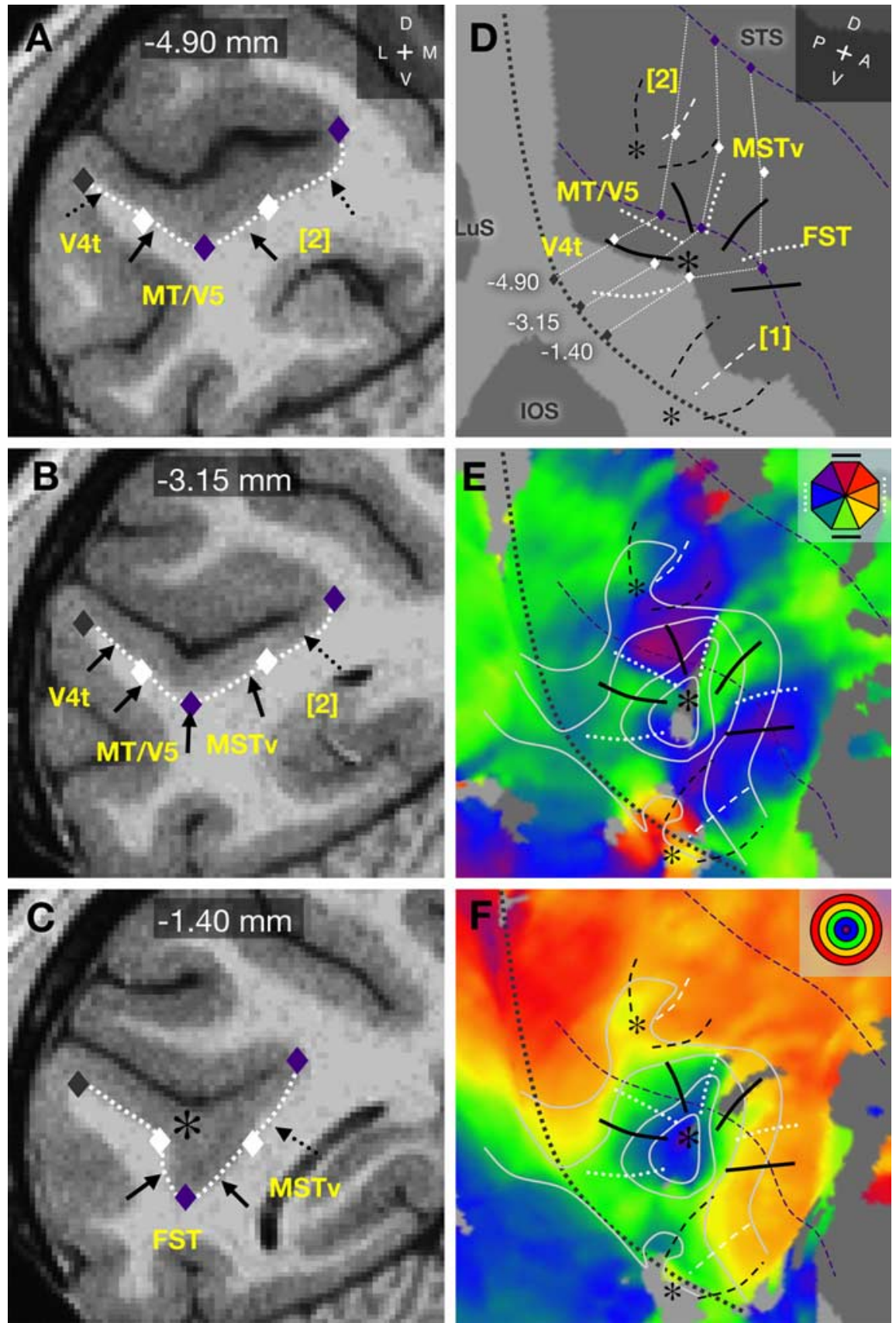

Figure 4. Anatomical location of polar angle and eccentricity representations in the MT/V5 cluster and surrounding areas; subject $\mathrm{CH}$. $A-C$, Three coronal sections (T1-weighted images) at $-4.90 \mathrm{~mm},-3.15$, and $-1.40 \mathrm{~mm}$ relative to the interaural plane. Indicated are the following: lip of the STS at the white- gray matter boundary (gray diamonds), posterior and anterior extent of the floor of the STS (purple diamonds), and intermediate locations (white diamonds). Solid arrows indicate the position of areal borders as defined by vertical meridian representations. Dotted arrows indicate uncertain locations of the most peripheral borders, given the restricted size of the stimuli used $\left(12^{\circ}\right.$ eccentricity). These anatomical landmarks are projected onto a flattened portion of the caudal STS in $\boldsymbol{D}$. Fine dotted white lines indicate the location of the coronal slices in steps of $1.75 \mathrm{~mm}$ (every fifth slice) that cover the MT/V5 cluster and surrounding area. The gray dotted line indicates the lip of the STS, and the dashed purple lines indicate the dorsal and ventral border of the floor of the STS. $E, F$, The polar angle and eccentricity maps with respect to the anatomical landmarks.

sphere (subject $\mathrm{CH}$, same data as in Fig. 2). Two consecutive cycles of lower- and upper-field representations can be observed if one moves along the curved lines (Fig. $3 B, C$ ). Further, Figure $3 A$ illustrates test-retest reproducibility and across-hemisphere symmetry. The data revealed excellent agreement for polar angle phase reversals (1) between two independently acquired datasets from the same monkey in experiments separated by $\sim 5.5$ months (open vs closed symbols) and (2) between two hemispheres in the same animal (upper vs lower traces). This topographic organization of MT/V5 and its satellites was consistently found in all four hemispheres imaged as shown by a test-retest analysis (supplemental Figs. S3-S6, available at www.jneurosci.org as supplemental material). In general, the observed functional layout resembles a pinwheel structure, exactly the hallmark of a field map cluster (Wandell et al., 2005).

In Figure 4, we show the detailed spatial relationship of the polar angle and eccentricity representations between a flattened reconstruction of the STS and the corresponding coronal sections through area MT/V5 and its satellites. The eccentricity and polar angle lines in the schematics of Figure $4 D-F$ were constructed based on a combination of polar angle, eccentricity, and field sign maps as described in supplemental Figures S3-S6 (available at www. jneurosci.org as supplemental material) and Materials and Methods. In Figure $4 A-C$, we indicated on three coronal T1weighted sections the position of several arbitrarily chosen anatomical landmarks at the boundary between gray and white matter-such as the ventral lip of the STS (gray diamonds), the boundaries of the floor of the STS (purple diamonds), and points in between (white diamonds). These landmarks were projected onto a two-dimensionally reconstructed portion of the STS as shown on the flat maps in Figure $4 D-F$. The same procedure was performed for all coronal sections covering this portion of the STS; hence, these flat maps show the exact location of the field maps with respect to the posterior lip of the STS (gray dotted line) and the dorsal and ventral limits of the floor of the fundus (purple dashed lines).

Based on the detailed anatomical location of the meridian representations (Fig. $4 E, F)$, we attribute the quarter-field and the three hemifield representations to areas V4t, MT/V5, ventral medial superior temporal area (MSTv), and fundus of superior temporal sulcus area (FST) (Desimone and Ungerleider, 1986; Komatsu and Wurtz, 1988; Andersen et al., 1990; Tanaka et al., 1993). We found weak evidence for an additional quarter-field representation of the upper visual field ventrally to V4t, which, based on the polar angle and field sign map, could be a complementary part of a hemifield at this location. At present, however, we lack further evidence from functional data for this part of the cortex and are not able to attribute this quadrant to a specific area. Therefore, we have assigned areas based on what is supported by literature, i.e., V4t is a quarter-field repre- 
sentation. To illustrate the quality of the fMRI signal changes within the MT/V5 cluster, we show session-averaged time courses of five selected voxels, which are located in the lower bank of the STS and which are confined to V4t and MT/V5 (Fig. 5). It is apparent from the time courses that the receptive field size of the neurons within these voxels is sufficiently small to allow reliable phase analyses since the signal returns to baseline between two periods of activations.

Supplemental Figures S3-S6 (available at www.jneurosci.org as supplemental material) show evidence of two additional central or near-central representations with associated field maps. One is located ventral (1) and another dorsal (2) relative to the MT/V5 cluster (see also Fig. 2). The dorsal near-central representation (2) is most obvious in subject TO, right hemisphere (supplemental Fig. S5, available at www.jneurosci.org as supplemental material), with minimum eccentricity values near $3^{\circ}$. It is associated with a hemifield map with perpendicular polar angle and eccentricity representations and can be seen in all tested hemispheres (supplemental Figs. S3-S5, available at www. jneurosci.org as supplemental material), except for the left hemisphere of subject TO (supplemental Fig. S6, available at www.jneurosci.org as supplemental material). The ventral central representation can be seen in all tested hemispheres with eccentricity values as low as $1^{\circ}$, which is the lowest eccentricity tested by the stimulus (supplemental Figs. S3-S6, available at www.jneurosci.org as supplemental material). It is associated with a hemifield in all hemispheres (supplemental Figs. S3-S5, available at www.jneurosci.org as supplemental material), except for the left hemisphere in subject TO (supplemental Fig. S6, available at www.jneurosci.org as supplemental material). This organization supports a dorsal and ventral field map with a full hemifield representation, distinct from the MT/V5 cluster.

The more dorsally located near-central representation [2] lacks the blue color-code in the eccentricity map, as one would expect for a true central visual field. However, the general circular structure of the eccentricity map and the associated hemifield representation suggest that it is a near-central, and not a midperipheral, representation. We attribute this effect to a combination of larger receptive field sizes in [2] compared with the foveal representation in the MT/V5 cluster and averaging effects due to a finite imaging resolution.

The apparent activation seen in the foveal representation of areas V1, V2, and V3 can be caused by surround inhibition beyond the actual stimulus leading to a negative BOLD signal (Brewer et al., 2002; Sereno and Tootell, 2005; Saygin and Sereno, 2008). In eccentricity measurements, this can lead to wrap around of the phase angle at the stimulus edge at low and high eccentricities, resulting in a continuation of the eccentricity map beyond this edge. This effect is more pronounced in areas with a large cortical magnification factor and relatively small receptive field sizes such as V1 and V2, and vanishes for small areas with larger receptive field sizes such as in the MT/V5 cluster. We have observed this effect in all four hemispheres near the fovea of the primary visual areas and indicated the approximate edge of the stimulus by a dashed-dotted gray line in Figure 2 and supplemental Figure S7 (available at www.jneurosci.org as supplemental material).

\section{Discussion}

High-field (7 T) fMRI in awake monkeys using accelerated imaging and slice-by-slice motion-correction algorithms allowed us to acquire high-resolution functional images $\left(0.42 \mathrm{~mm}^{3}\right.$ voxels $)$ with little distortion relative to the anatomy. Thanks to these technical advances, we could identify three complete hemifield and one quarter-field representation surrounding a foveal representation at the location of MT/V5 and its satellites. These data indicate the existence of a field map cluster in the posterior portion of the STS.

Although several motion-selective satellites of monkey area MT/V5 have been described, including MSTd (dorsal MST), MSTv, FST, STPa (anterior superior temporal polysensory area), and LST (lower superior temporal region) (Desimone and Ungerleider, 1986; Mikami et al., 1986; Saito et al., 1986; Maunsell and Van-Essen, 1987; Komatsu and Wurtz, 1988; Duffy and 
Wurtz, 1991; Tanaka et al., 1993; Nelissen et al., 2006), their extent and exact partitioning scheme has remained elusive despite considerable efforts to map these areas. Contrary to electrophysiological mapping, fMRI has the advantage of a relatively large field of view, avoiding the need to interpolate data from many experiments. So far, monkey fMRI has been used successfully to map the retinotopic (Brewer et al., 2002; Fize et al., 2003), functional (Sereno et al., 2002; Tsao et al., 2003; Nelissen et al., 2006; Vanduffel et al., 2001, 2002), and anatomical (Tolias et al., 2005; Ekstrom et al., 2008) properties of the monkey area MT/V5 and surrounding regions. However, none of these studies has had sufficient spatial resolution to resolve the fine topographic structure of these motion-selective areas within the STS. Because of the overrepresentation of the fovea in many cortical areas, polar angle representations change much faster than eccentricity as a function of cortical distance-particularly at low eccentricity representations. Therefore, low-spatial-resolution imaging techniques have hampered the detection of polar angle maps in small extrastriate visual areas, some of which are only a few millimeters wide-such as area V4t. In the present study, we had to turn to high-field high-resolution fMRI to investigate in detail the retinotopic organization of the posterior portion of the STS in the monkey.

Can the updated topographic map of MT/V5 and its satellites (Fig. 4D) be reconciled with previous information derived from electrophysiology? Most published data [for a summary, see Komatsu and Wurtz (1988), their Fig. 13] show a foveal representation at the most ventral extent of MT/V5, and/or the most lateral portion of MSTl (lateral MST), and/or the most dorsal portion of FST (Desimone and Ungerleider, 1986; Maunsell and Van Essen, 1987; Komatsu and Wurtz, 1988; Nelissen et al., 2006). The current finding that this foveal representation actually corresponds to the center of a cluster of four field maps significantly expands upon those electrophysiological results as well as upon anatomical connectivity data [see Andersen et al. (1990), their Fig. 10].

The fMRI experiments also indicated the existence of two additional hemifield representations [1] and [2] neighboring the MT/V5 cluster. The central representation [1] is consistently found ventrally to areas V4t and FST and anterior to the foveal confluence of V1 through V4. Above this location, the alternating sequence of polar angle representations within the MT/V5 cluster itself is interrupted (Fig. $3 B, C$ ). This gap (i.e., the cortical region between $\mathrm{a}$ and $\mathrm{b}$, and $\mathrm{c}$ and $\mathrm{d}$ in Fig. $3 B, C$ ) coincides with $\mathrm{a}$ low-eccentricity ridge (green pseudocolor) between the MT/V5 cluster and foveal representations of more caudal visual areas (Fig. $4 F$ ). The eccentricity lines of FST continue anterior to this gap. The polar angle values are consistent with a separate hemifield but are not associated with those of the MT/V5 cluster. We attribute this region to a field map, which could correspond to PITd (dorsal posterior inferotemporal area) (Boussaoud et al., 1991; Distler et al., 1993). More generally, such "gaps" in the expected circular eccentricity representation may be a fundamental property of field map clusters at locations where two neighboring clusters touch. Because of spatial constraints, it is to be expected that at such connecting points, eccentricity lines continue from the one to the other cluster exactly as we observed. Therefore, as argued above, central representation [1] may be the center of an adjoining more ventrally located cluster, which was only partially covered by the slices in the present experiment. Moreover, careful inspection of the VO1-VO2 data from Wandell et al. (2007) indicates that a visual field map cluster may be

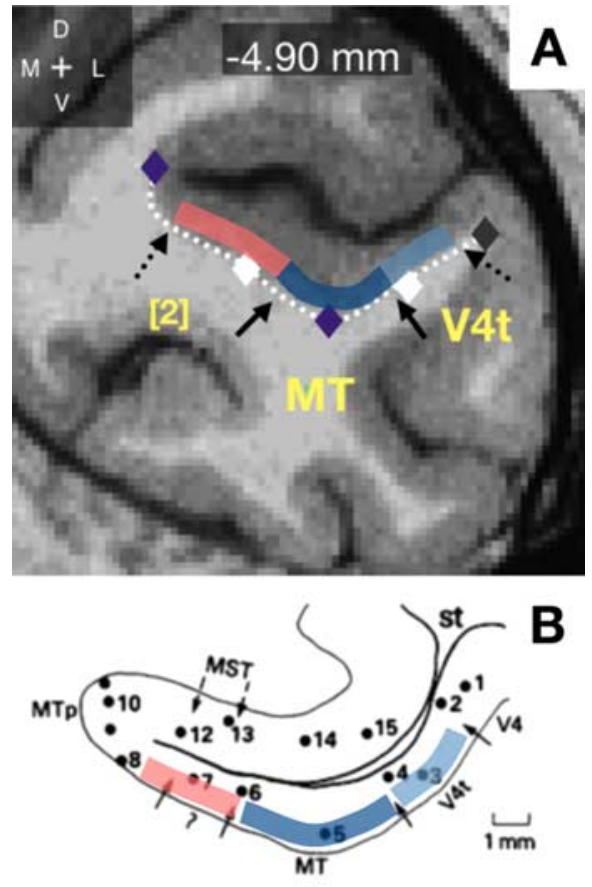

Desimone \& Ungerleider 1986

Figure 6. Comparison of current $\mathrm{fMRI}$ results, subject $\mathrm{CH}$, right hemisphere, and electrophysiology results from Desimone and Ungerleider (1986). $A$, Location of area borders based on fMRI result in the current study (Fig. $4 A$ ). The image is left-right reversed compared with Figure 4 to allow a better comparison with electrophysiology results. $\boldsymbol{B}$, Location of area borders as taken with permission from Desimone and Ungerleider (1986) (their Fig. 10).

better described as a partial rather than a complete "pinwheel" map, unlike the suggestion in their Figure $9 A$.

A direct comparison of the topographic maps with previous electrophysiological data is shown in Figure $6 \mathrm{~A}$, where the borders of areas V4t, MT/V5, and the newly found hemifield [2] are projected onto a coronal slice (Fig. 6B) at a similar position as presented by Desimone and Ungerleider (1986). The two modalities show good agreement for areas V4t and MT/V5. The electrophysiological recordings further suggested the existence of a region "MTp" (MT, peripheral visual field) medial to area MT/ V5, which represents the far periphery of the visual field and is thought to be an architectonically distinguishable subdivision of MT/V5. At the corresponding location, we found a representation of a hemifield [2] with a relatively strong periphery bias and a near-central representation (see above). When considering the exact location of the electrophysiological recordings (Desimone and Ungerleider, 1986) there is a surprisingly good agreement between the localization of area MTp and the present fMRI-based field map [2] (Fig. 4D). However, our data suggest that this field map also contains a near-central representation. A possible interpretation of this result is that the hemifield representation [2] observed in the present experiment is completely distinct from MT.

Previous studies indicated a different topographic organization of MT/V5 and surrounding areas in the New World monkey compared with the present results in Old World monkeys. In the former, area MT/V5 is surrounded by a quarter-field (MT crescent) rather than multiple hemifield representations (Allman and Kaas, 1974). Moreover, MST in the New World monkey does not share a foveal representation with MT/V5. Such layout resembles the well known topographic organization of areas V1, V2, V3, 
and V4 in both Old and New World monkeys. Rosa and Tweedale (2005) suggested that MT/V5 is a primordial "core field" similar to V1 that emerges early during development and that is evolutionary older than "noncore" areas. This fundamental area serves as an anchor around which noncore areas emerge as quarter-field representations. The layout of the cluster as we observed here, however, does not single out particular areas as core or noncore areas. Hence the MT/V5-MTc (MT crescent) organization in New World monkeys, which resembles a nucleus with a quarter-field "shell," may be a precursor of the pinwheel-like cluster observed in Old World monkeys. This may suggest that the type of pinwheel-like MT/V5 clusters only developed after the common ancestor of New and Old World monkeys $\sim 35$ million years ago (Schrago and Russo, 2003).

In summary, high-field monkey fMRI data of the posterior STS revealed three full and one half representation of the contralateral hemifield, which surround a single foveal representation and share a continuous eccentricity map spanning a three-quarter circle. A schematic overview of the topographic organization of the MT/V5 cluster in all tested hemispheres is shown in Figure 7. The functional organization that emerges reconciles previous often contradictory maps of the STS that are mainly based on electrophysiological recordings (Komatsu and Wurtz, 1988) and anatomical tractography data (Andersen et al., 1990). The fMRI results fit surprisingly well with a recent model of cortical functional organization based on field map clusters (Wandell et al., 2005, 2007). Moreover, the present findings indicate that such clusters are not an exclusive property of the ventral stream but exist in prototypical dorsal stream regions. Exactly as predicted theoretically, we show that clusters tie together field maps known to be involved in specific perceptual functions-in this case visual motion processing (Zeki, 1974; Desimone and Ungerleider, 1986; Maunsell and Van Essen, 1987; Komatsu and Wurtz, 1988; Nelissen et al., 2006). Furthermore, we show that clusters are not human specific and are also present in Old World monkeys. We conclude that visual field map clusters are evolutionarily preserved and, hence, may be a fundamental organizational principle of the Old World primate visual cortex. Therefore, we predict that improving the spatial resolution of human fMRI experiments will reveal a similar functional organization in human MT/V5+ as was observed here in the macaque.

\section{References}

Allman JM (1999) Evolving brains. New York: Scientific American Library/W. H. Freeman.

Allman JM, Kaas JH (1974) A crescent-shaped cortical visual area surrounding the middle temporal area (MT) in the owl monkey (Aotus trivirgatus). Brain Res 81:199-213.

Andersen RA, Asanuma C, Essick G, Siegel RM (1990) Corticocortical connections of anatomically and physiologically defined subdivisions within the inferior parietal lobule. J Comp Neurol 296:65-113.
Bonhoeffer T, Grinvald A (1991) Iso-orientation domains in cat visual cortex are arranged in pinwheel-like patterns. Nature 353:429-431.

Boussaoud D, Desimone R, Ungerleider LG (1991) Visual topography of area TEO in the macaque. J Comp Neurol 306:554-575.

Brewer AA, Press WA, Logothetis NK, Wandell BA (2002) Visual areas in macaque cortex measured using functional magnetic resonance imaging. J Neurosci 22:10416-10426.

Brewer AA, Liu J, Wade AR, Wandell BA (2005) Visual field maps and stimulus selectivity in human ventral occipital cortex. Nat Neurosci 8:1102-1109.

Chklovskii DB, Koulakov AA (2004) Maps in the brain: what can we learn from them? Annu Rev Neurosci 27:369-392.

Desimone R, Ungerleider LG (1986) Multiple visual areas in the caudal superior temporal sulcus of the macaque. J Comp Neurol 248:164-189.

Distler C, Boussaoud D, Desimone R, Ungerleider LG (1993) Cortical connections of inferior temporal area TEO in macaque monkeys. J Comp Neurol 334:125-150.

Duffy CJ, Wurtz RH (1991) Sensitivity of MST neurons to optic flow stimuli. I. A continum of response selectivity to large-field stimuli. J Neurophysiol 65:1329-1345.

Dumoulin SO, Wandell BA (2008) Population receptive field estimates in human visual cortex. Neuroimage 39:647-660.

Ekstrom LB, Roelfsema PR, Arsenault JT, Bonmassar G, Vanduffel W (2008) Bottom-up dependent gating of frontal signals in early visual cortex. Science 321:414-417.

Felleman DJ, Van Essen DC (1991) Distributed hierarchical processing in the primate cerebral cortex. Cereb Cortex 1:1-47.

Fischl B, Sereno MI, Dale AM (1999) Cortical surface-based analysis. II: Inflation, flattening, and a surface-based coordinate system. Neuroimage 9:195-207.

Fize D, Vanduffel W, Nelissen K, Denys K, Chef d'Hotel C, Faugeras O, Orban 
GA (2003) The retinotopic organization of primate dorsal V4 and surrounding areas: a functional magnetic resonance imaging study in awake monkeys. J Neurosci 23:7395-7406.

Goense JB, Ku SP, Merkle H, Tolias AS, Logothetis NK (2008) fMRI of the temporal lobe of the awake monkey at 7 T. Neuroimage 39:1081-1093.

Goodale MA, Milner AD (1992) Separate visual pathways for perception and action. Trends Neurosci 15:20-25.

Hasson U, Levy I, Behrmann M, Hendler T, Malach R (2002) Eccentricity bias as an organizing principle for human high-order object areas. Neuron 34:479-490.

Komatsu H, Wurtz RH (1988) Relation of cortical areas MT and MST to pursuit eye movements. I. Localization and visual properties of neurons. J Neurophysiol 60:580-603.

Larsson J, Heeger DJ (2006) Two retinotopic visual areas in human lateral occipital cortex. J Neurosci 26:13128-13142.

Leite FP, Mandeville JB (2006) Characterization of event-related designs using BOLD and IRON fMRI. Neuroimage 29:901-909.

Madore B, Glover GH, Pelc NJ (1999) Unaliasing by Fourier-encoding the overlaps using the temporal dimension (UNFOLD), applied to cardiac imaging and fMRI. Magn Reson Med 42:813-828.

Maunsell JHR, Van Essen DC (1987) Topographic organization of the middle temporal visual area in the macaque monkey: representational biases and the relationship to callosal connections and myeloarchitectonic boundaries. J Comp Neurol 266:535-555.

Mikami A, Newsome WT, Wurtz RH (1986) Motion selectivity in macaque visual cortex. I. Mechanisms of direction and speed selectivity in extrastriate area MT. J Neurophysiol 55:1308-1327.

Mitchison G (1991) Neuronal branching patterns and the economy of cortical wiring. Proc Biol Sci 245:151-158.

Nelissen K, Vanduffel W, Orban GA (2006) Charting the lower superior temporal region, a new motion-sensitive region in monkey superior temporal sulcus. J Neurosci 26:5929-5947.

Pruessmann KP, Weiger M, Scheidegger MB, Boesiger P (1999) SENSE: sensitivity encoding for fast MRI. Magn Reson Med 42:952-962.

Rosa MG, Tweedale R (2005) Brain maps, great and small: lessons from comparative studies of primate visual cortical organization. Philos Trans R Soc Lond B Biol Sci 360:665-691.

Saito H, Yukie M, Tanaka K, Hikosaka K, Fukada Y, Iwai E (1986) Integration of direction signals of image motion in the superior temporal sulcus of the macaque monkey. J Neurosci 6:145-157.

Saygin AP, Sereno MI (2008) Retinotopy and attention in human occipital, temporal, parietal, and frontal cortex. Cereb Cortex 18:2158-2168.
Schrago CG, Russo CA (2003) Timing the origin of New World monkeys. Mol Biol Evol 20:1620-1625.

Sereno ME, Trinath T, Augath M, Logothetis NK (2002) Threedimensional shape representation in monkey cortex. Neuron 33:635-652.

Sereno MI, Tootell RB (2005) From monkeys to humans: what do we now know about brain homologies? Curr Opin Neurobiol 15:135-144.

Sereno MI, Dale AM, Reppas JB, Kwong KK, Belliveau JW, Brady TJ, Rosen BR, Tootell RBH (1995) Borders of multiple visual areas in humans revealed by functional MRI. Science 268:889-893.

Smith AT, Singh KD, Williams AL, Greenlee MW (2001) Estimating receptive field size from fMRI data in human striate and extrastriate visual cortex. Cereb Cortex 11:1182-1190.

Tanaka K, Sugita Y, Moriya M, Saito H (1993) Analysis of object motion in the ventral part of the medial superior temporal area of the macaque visual cortex. J Neurophysiol 69:128-142.

Tolias AS, Sultan F, Augath M, Oeltermann A, Tehovnik EJ, Schiller PH, Logothetis NK (2005) Mapping cortical activity elicited with electrical microstimulation using FMRI in the macaque. Neuron 48:901-911.

Tsao DY, Vanduffel W, Sasaki Y, Fize D, Knutsen TA, Mandeville JB, Wald LL, Dale AM, Rosen BR, Van Essen DC, Livingstone MS, Orban GA, Tootell RB (2003) Stereopsis activates V3A and caudal intraparietal areas in macaques and humans. Neuron 39:555-568.

Ungerleider LG, Mishkin M (1982) Two cortical visual systems. In: The analysis of visual behavior (Ingle DJ, Mansfield RJW, Goodale MS, eds), pp 549-586. Cambridge, MA: MIT.

Vanduffel W, Fize D, Mandeville JB, Nelissen K, Van Hecke P, Rosen BR, Tootell RB, Orban GA (2001) Visual motion processing investigated using contrast agent-enhanced fMRI in awake behaving monkeys. Neuron 32:565-577.

Vanduffel W, Fize D, Peuskens H, Denys K, Sunaert S, Todd JT, Orban GA (2002) Extracting 3D from motion: differences in human and monkey intraparietal cortex. Science 298:413-415.

Wandell BA, Brewer AA, Dougherty RF (2005) Visual field map clusters in human cortex. Philos Trans R Soc Lond B Biol Sci 360:693-707.

Wandell BA, Dumoulin SO, Brewer AA (2007) Visual field maps in human cortex. Neuron 56:366-383.

Zeki SM (1974) Functional organization of a visual area in the posterior bank of the superior temporal sulcus of the rhesus monkey. J Physiol 236:549-573. 